Christine Funke

\section{Einführung}

Erkältungskrankheiten manifestieren sich in Zeiten, die sich durch den Temperaturwechsel von warm zu kalt auszeichnen. Arzneipflanzen bewältigen diesen aus eigener Kraft und können daher mit ihrer lebendigen Heilessenz einen erkälteten Körper ohne Nebenwirkungen auf ein gesundes Niveau transformieren. Die Arzneimittelherstellung, die Transformationsprozesse in dieser Art anwendet, ist die Spagyrik.

Spagyrisch pflanzliche Essenzen sind dank ihres aufwendigen Herstellungsprozesses frei von Gift- und Ballaststoffen, weisen einen Alkoholgehalt von $18-20 \%$ auf und können bei allen Altersgruppen angewendet werden. Dank der Resorption über die Mundschleimhaut wirken sie rasch und belasten die Leber nicht.

Wie kann Spagyrik bei

Erkältungskrankheiten helfen?

Erkältungskrankheiten zeichnen sich durch Unterkühlung, Entzündungen sowie Viren- und Bakterienbefall aus. Spagyrische Essenzen wirken dank feinstofflich herausgearbeiteter Heilessenz tiefgreifend, indem sie den unterkühlten Körper wärmen und nach ihrem Wirkungscharakter antiphlogistisch, antiviral, antibiotisch, abwehrsteigernd, reizmildernd (Husten) und schleimhautsanierend regenerieren.

*Nach einem Beitrag zur 3. Jahrestagung der Schweizerischen Arbeitsgemeinschaft für Perinatale Pharmakologie (SAPP) vom 9.12.2010.

\section{Fortbildung}

Schweiz Z Ganzheitsmed 2011;23:331

DOI: 10.1159/000334361

\title{
Echinacea, Granatapfel und Co - eine Einführung in die spagyrische Behandlung von Erkältungskrankheiten*
}

Spagyrik ist eine uralte Herstellungsmethode, die auf der Naturphilosophie Alchemie gründet und die die Laborprozesse Gärung, Destillation und Veraschung umfasst. Diese Prozesse dauern pro Essenzherstellung mindestens 3 Monate. Dank dieser aufwendig langen Herstellung gewinnt man die reine pflanzliche Heilessenz, die frei von Ballast- und Giftstoffen ist.

\section{Ausgewählte Arzneipflanzen}

Echinacea purpurea (Purpur-Sonnenhut, Abb. 1) wirkt antiviral und immunmodulierend. Bei Attacken von Grippeviren hat sich Punica granatum (Granatapfel, Abb. 2) bewährt, und bei langwierigem Virusbefall hilft Vincetoxicum hirundinarium (Schwalbenwurz). Bei fortgeschrittener Erkältung mit bakteriellem Befall wirkt Tropaeolum majus (Große Kapuzinerkresse) antibiotisch, insbesondere wenn Antibiotikaresistenz besteht. Hyperreagibile Schleimhäute des Atemtrakts beruhigt der Papaver rhoeas (Klatschmohn), zähflüssigen Bronchialschleim löst Primula veris (Echte Schlüsselblume) und unaufhörlichen Reizhusten stoppt Drosera rotundifolia (Rundblättriger Sonnentau). Für nachhaltige Schleimhautsanierung hat sich bei allen Altersklassen Sambucus nigra (Schwarzer Holunder) bewährt, der lang anhaltend wärmt und saniert.

\section{Dosierung spagyrischer Essenzen}

Kinder: 2- bis 3-mal täglich 2 Hübe; Säuglinge: 1-3 Hübe in den täglichen «Teeschoppen»; Erwachsene: akut 5- bis 10-mal 1-2 Hübe, chronisch 3- bis 5-mal täglich 2 Hübe.

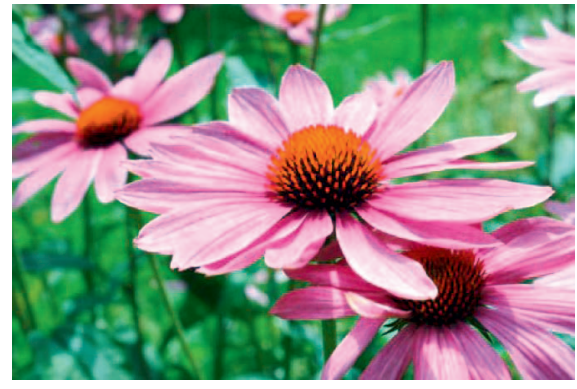

Abb. 1. Echinacea purpurea (PurpurSonnenhut).

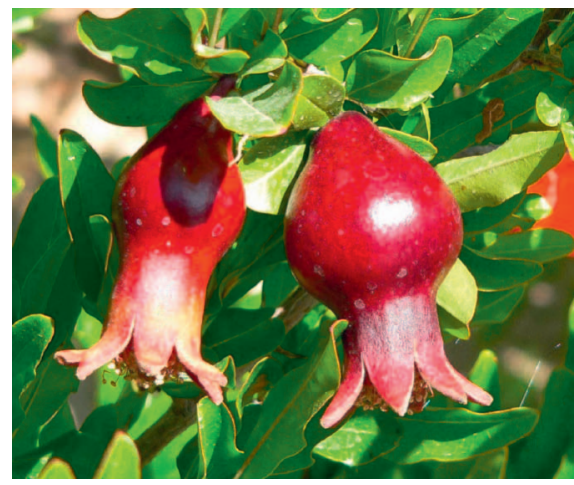

Abb. 2. Punica granatum (Granatapfel).

\section{Zusammenfassung}

Spagyrika wirken bei Erkältungserkrankungen ohne Nebenwirkungen und weisen einen schnellen Wirkungseintritt auf. Als Mittel erster Wahl wirken sie tiefgreifend auf die körpereigenen Regulationssysteme, sodass Selbstheilungskräfte aktiviert werden. Spagyrische Essenzen wirken gegen Erkältungserkrankungen immunmodulierend, antiviral, antiphlogistisch, antibiotisch oder hyperämisierend (durchwärmend) auf Schleimhäute. Erfahrungsmedizinisch bewährt haben sich z.B. E. purpurea als Immunmodulator, P. granatum als Antigrippemittel und V. hirundinarium bei viralem Befall.
(๑) 2011 S. Karger GmbH, Freiburg

Accessible online at: www.karger.com/szg
Christine Funke

Eidg. dipl. Apothekerin, FPH Offizin und FH Phytotherapie Spagyros AG

Tannackerstrasse 7, 3073 Gümligen, Schweiz

christine.funke@spagyros.ch 